\title{
GYMNOPILUS TUXTLENSIS (BASIDIOMYCETES, AGARICALES), ESPECIE TROPICAL CONOCIDA DE MÉXICO Y AMÉRICA DEL SUR
}

\author{
Laura Guzmán-Dávalos ${ }^{1}$, María de Jesús Herrera ${ }^{1}$, Beatriz E. Cardona ${ }^{2}$ y \\ YAMILLÉ SALDARRIAGA ${ }^{2}$ \\ ${ }^{1}$ Universidad de Guadalajara, Instituto de Botánica, Departamento de Botánica y \\ Zoología, Apdo. postal 1-139, 45101 Zapopan, Jalisco, México \\ lguzman@cucba.udg.mx \\ ${ }^{2}$ Universidad de Antioquia, Corporación de Patologías Tropicales, \\ Apdo. postal 1226, Medellín, Colombia
}

\section{RESUMEN}

Gymnopilus tuxtlensis fue descrito a partir de ejemplares recolectados en la región de Los Tuxtlas, Veracruz, México. En este trabajo se estudiaron más de 20 materiales recolectados recientemente o depositados en herbarios, con lo que se amplía su distribución conocida en México al registrarla de Jalisco, y se cita por primera vez de América del Sur, con base en especímenes de Colombia y Venezuela. Esta especie crece en regiones tropicales y es muy característica por su basidioma pequeño, de color amarillo-mostaza, con una base estrigosa, por presentar pleurocistidios grandes y de forma característica, y queilocistidios más pequeños.

Palabras clave: Agaricales, Basidiomycetes, Colombia, Gymnopilus tuxtlensis, Jalisco, pleurocistidios, taxonomía, Venezuela, Veracruz.

\begin{abstract}
Gymnopilus tuxtlensis was described on the basis of specimens from Los Tuxtlas region, Veracruz, Mexico. In this work, more than 20 specimens either recently collected or deposited in several herbaria were studied. These specimens document a wider distribution of the species in Mexico (Jalisco), and its first record from South America, based on collections from Colombia and Venezuela. This species grows in tropical regions and is very characteristic because of its small, mustard-yellow basidioma, with a strigose base, long and characteristic pleurocystidia and smaller cheilocystidia.
\end{abstract}

Key words: Agaricales, Basidiomycetes, Colombia, Gymnopilus tuxtlensis, Jalisco, pleurocystidia, taxonomy, Venezuela, Veracruz. 


\section{INTRODUCCIÓN}

El género Gymnopilus, al igual que otros grupos de hongos, ha sido poco estudiado en los trópicos, y son relativamente escasas las especies que se conocen de estas áreas. Aunque se creía que tiene una distribución principalmente en regiones templadas, es muy probable que sea rico en número de componentes en comarcas de clima cálido, como lo hizo ver Guzmán-Dávalos (2002) para el caso de México.

Gymnopilus tuxtlensis fue descrita con base en ejemplares recolectados en una zona tropical, en la región de Los Tuxtlas (Guzmán-Dávalos, 1994) y se pensaba que era exclusiva de México (Guzmán-Dávalos, 2002). Al revisar materiales depositados en herbarios y con recolectas recientes, se le encontró creciendo en otros sectores de México y en dos países sudamericanos, indicando que la distribución de esta especie es mucho más amplia, y se extiende por ahora a América tropical.

\section{MATERIALES Y MÉTODOS}

Se tomaron datos macromorfológicos de los especímenes frescos; en el caso de materiales de herbario éstos se registraron a partir de la interpretación de lo examinado en los ejemplares deshidratados. Las observaciones microscópicas se realizaron en montajes en hidróxido de potasio $(\mathrm{KOH})$ a $5 \%$, en reactivo de Melzer para la reacción dextrinoide, en azul de cresil para la reacción metacromática y en azul de algodón para la cianofilia de las basidiosporas. La medida de las esporas incluye la ornamentación pero no el apéndice hilar. Se midieron al menos 20 esporas por ejemplar, y se calculó el coeficiente Q (longitud entre ancho). Los dibujos de las estructuras microscópicas se hicieron directamente o con la ayuda de un tubo de dibujo. Los acrónimos de los herbarios se dan con base en Holmgren et al. (1990). Las claves de los colores, cuando se indican, son de Kornerup y Wanscher (1978).

\section{RESULTADOS Y DISCUSIÓN}

Gymnopilus tuxtlensis Guzm.-Dáv., Mycotaxon 50: 345, 1994 (como “tuxtlense”) Figs. 1 y 2

Píleo de 3-24 (-30) mm de diám., convexo, campanulado, plano-convexo, planocampanulado a plano con depresión central o cóncavo, mamiforme a umbilicado, 
glabro a levemente fibriloso-algodonoso, liso, de color amarillo pálido (3A3, 3A4), amarillo (3A8), amarillo-mostaza, amarillo-oro (5B7), amarillo-anaranjado (5A7), anaranjado con tonos mostaza, café-mostaza, café-amarillento u ocre-amarillo (5C7), café-anaranjado-grisáceo (5B6), higrófano; margen recto, entero o estriado hasta el mamelón, estrías por transparencia, o muy marcadas como canales. Contexto muy delgado, blanquecino, amarillento o amarillo. Láminas adheridas a sinuadas, subdistantes, segmentiformes, delgadas, borde liso, de color amarillo-pálido (3A3), amarillo (4A5), amarillo-mostaza, amarillo-anaranjado (5A7), a anaranjado-ocre (5B8), finalmente ferruginosas. Estípite de 5-40 x 0.5-2 mm, central a excéntrico, uniforme o ensanchado hacia arriba o hacia la base, cilíndrico, pruinoso a fibriloso en el ápice, el resto glabro o fibriloso, hueco, amarillo pálido (3A3), amarillo (4A5), o de color café-amarillento hacia la base, se mancha de color café-anaranjado o rojizo; base del estípite estrigosa en la unión con el sustrato, con abundante micelio o pelos rígidos, blanquecinos, amarillos o de color amarillo-mostaza; rizomorfos amarillos presentes entre la madera en algunos casos. Velo superior, muy delgado, evanescente, en la mayoría de los casos no observado. Olor fúngico, ligeramente dulce, farináceo o en ocasiones desagradable; sabor amargo muy fuerte. $\mathrm{KOH}$ tiñe el píleo de color café-rojizo.

Basidiosporas de 7-9 (-10.5) x (4-) 4.5-5.5 (-6) $\mu \mathrm{m}, \mathrm{Q}=1.4-1.8$ (-2), elipsoides a alongadas; ápice obtuso, subagudo o en ocasiones cortamente truncado; pared subgruesa, verrugosa, verrugas medianas a grandes; sin poro germinal, con placa (en la mayoría poco evidente), con leve depresión suprahilar; de color café-amarillento o café-anaranjado, dextrinoides, cianófilas, no metacromáticas (Fig. 1A). Basidios de 12-28 (-31) x 5-9.5 (-10) $\mu \mathrm{m}$, claviformes, con o sin constricción central, bi a tetraspóricos, hialinos y algunos con contenido de color café-amarillento o caféferruginoso; esterigmas de 2.5-7 $\mu \mathrm{m}$ de long. (Fig. 1C). Basidiolos de 11-23 x 5$10 \mu \mathrm{m}$, espatulados, claviformes, inflados, ventricosos, hialinos (Figs. 1I, J). Pleurocistidios de dos tipos: 1) de 11-24 (-27) x 4-9 $\mu \mathrm{m}$, ventricosos a cilíndricoventricosos, hialinos, algunos con contenido de color café-ferruginoso, muy escasos, y 2) de 30-60 x 8-17 $\mu \mathrm{m}$, ápice de 2.4-8.5 $\mu \mathrm{m}$, fusiformes, lageniformes, utriformes, ápice obtuso, subcapitado, capitado, submoniliforme o mucronado, pared delgada y en ocasiones engrosada hacia el ápice, amarillentos, con o sin contenido granuloso de color café-amarillento; escasos o abundantes, pero muy evidentes (Figs. 1B, D, F). Queilocistidios de 14-25 (-27.5) x 5.6-11.5 $\mu \mathrm{m}$, ápice de 2.4-9 $\mu \mathrm{m}$, utriformes, cortamente lageniformes, espatulados, ápice obtuso, pared delgada, hialinos o amarillentos, escasos (Figs. 1E, G, K). Borde heteromorfo con basidios, basidiolos y queilocistidios. Trama himenófora subparalela, hifas de 4-17.6 $\mu \mathrm{m}$ de diám., septadas, 


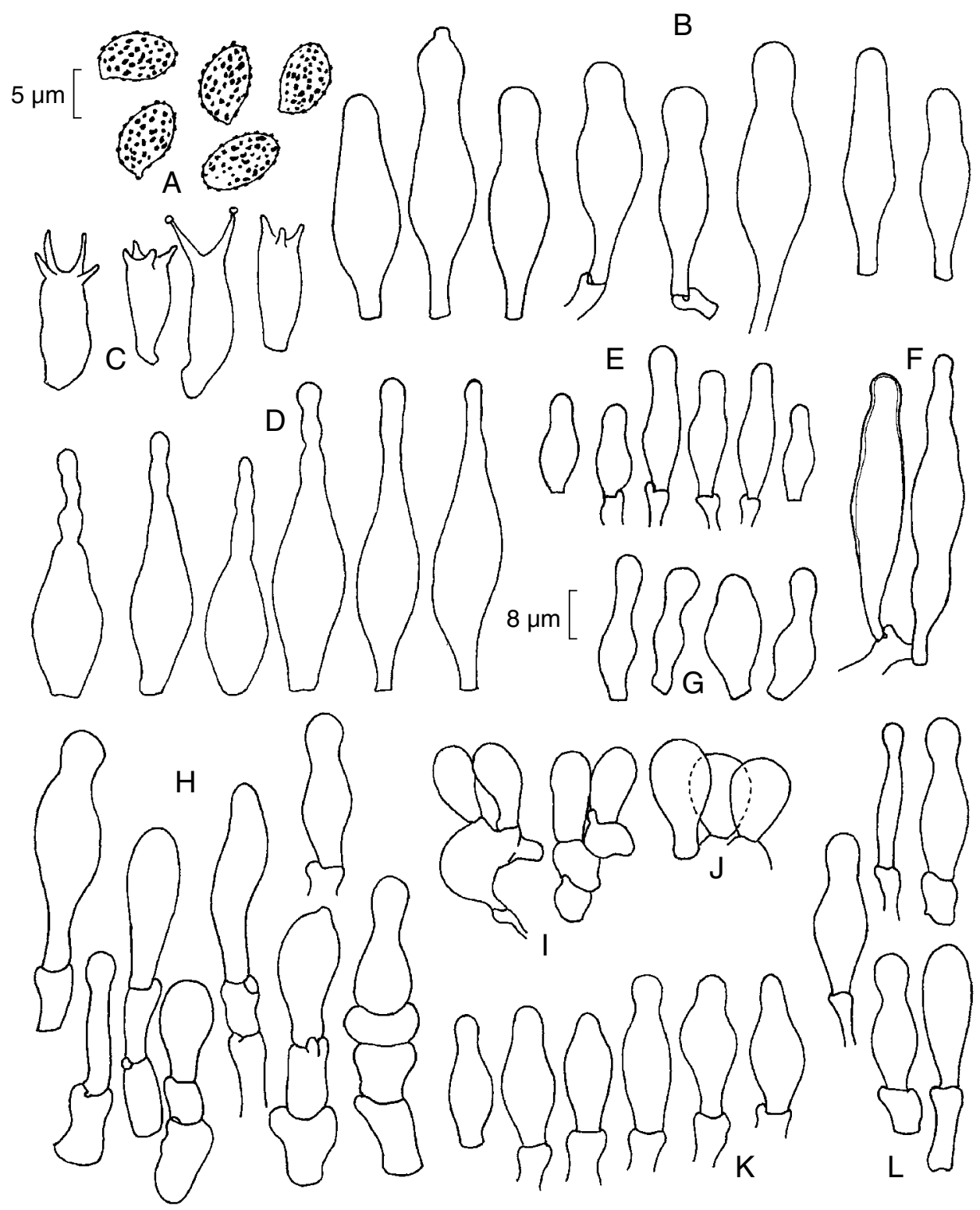

Fig. 1. Gymnopilus tuxtlensis. A: esporas; B: pleurocistidios; C: basidios; D: pleurocistidios, E: queilocistidios, F: pleurocistidios; G: queilocistidios; H: caulocistidios; I: subhimenio celular y basidiolos; J: basidiolos; K: queilocistidios, L: caulocistidios. A, C, F, G, I y J de GuzmánDávalos 9492; B, H y K de Ovrebo 1182-A; D, E y L de Guzmán-Dávalos 7674. Escala todos $=8 \mu \mathrm{m}$, excepto $\mathrm{A}=5 \mu \mathrm{m}$. 
con fíbulas, pared delgada, subgruesa a gruesa, amarillentas. Subhimenio celular (Fig. 1I). Trama del píleo radial, hifas de pared delgada a subgruesa, amarillentas. Pileipellis un cutis, con hifas de 4-13.5 $\mu \mathrm{m}$ de diám., postradas, septadas, con fíbulas, pared delgada a subgruesa, amarillentas o de color café-anaranjado, algunas con incrustaciones de pigmento en la pared en forma irregular, en pocas en bandas. Pileocistidios ausentes. Caulocistidios de 16-40 x 3-13 $\mu \mathrm{m}$, ápice de 3-9 $\mu \mathrm{m}$, cilíndricos, claviformes, lageniformes, subfusiformes, utriformes, con ápice obtuso, subcapitado o capitado, pared delgada, hialinos o amarillentos, a veces en cadenas cortas con hifas cortas o elementos cuadrangulares, dispuestos en fascículos en el ápice del estípite, escasos en la parte media, ausentes en la base (Figs. 1H, L). Hifas del estípite con pigmento incrustado en sus paredes en bandas. Hifas oleíferas presentes en todo el basidioma. En la mayoría de los casos los fragmentos de láminas liberan pigmento amarillento en $\mathrm{KOH}$.

Hábitat. Gregario a subcespitoso, sobre madera podrida, en bosque tropical perennifolio, bosque tropical caducifolio y en bosque de pino-encino en zona tropical (Fig. 2).

Material estudiado. COLOMBIA: Departamento de Antioquia, municipio de San Luis, Parque Natural Río Claro, octubre 9, 1985, alt 400 m, L. Velásquez 4 (HUA); 145 km al SE de Medellín, octubre 9, 1985, G. García 14 (HUA). MÉXICO: Estado de Jalisco, municipio de Puerto Vallarta, km 201 carretera Mismaloya-El Tuito, octubre 16, 1998, alt. 80 m, L. Guzmán-Dávalos 7668-A, 7668-B (IBUG); municipio de Cabo Corrientes, $3.3 \mathrm{~km}$ al S de Puente Las Cañadas, km 179 carretera Mismaloya-El Tuito, octubre 16, 1998, alt. 730-800 m, L. Guzmán-Dávalos 7670, 7674, 7675, 7693 (IBUG); 9.8 km brecha El Tuito a Aquiles Serdán, septiembre 13, 2004, alt. 570 m, M. Herrera 1146, L. Guzmán-Dávalos 9492 (IBUG); municipio de San Sebastián del Oeste, cerca del pueblo de San Sebastián del Oeste, septiembre 17, 1995, Y. S. Rubio s.n. (HUA, IBUG). Estado de Veracruz, municipio de San Andrés Tuxtla, carretera Catemaco a Montepío, Estación Biológica de Los Tuxtlas de la Universidad Nacional Autónoma de México, julio 26, 1981, G. Guzmán 19671 (XAL-Holotipo); Jardín Botánico, julio 26, 1981, G. Guzmán 19798, 19812, 19830 (XAL-Paratipos); Vigía 5, julio 28, 2004, O. Rodríguez 2613 (IBUG); Vereda Norte, camino a Laguna Escondida, julio 27, 2004, alt $130 \mathrm{~m}, M$. R. SánchezJácome 1040 (IBUG); municipio de Catemaco, alrededor de la Embotelladora de Coyame, E. Pérez-Silva obs. 95 (MEXU-19425); a $10 \mathrm{~km}$ entre Catemaco y Montepío, julio 10, 1977, J. Pérez-Ortiz 636 (ENCB). VENEZUELA: Llanos de 


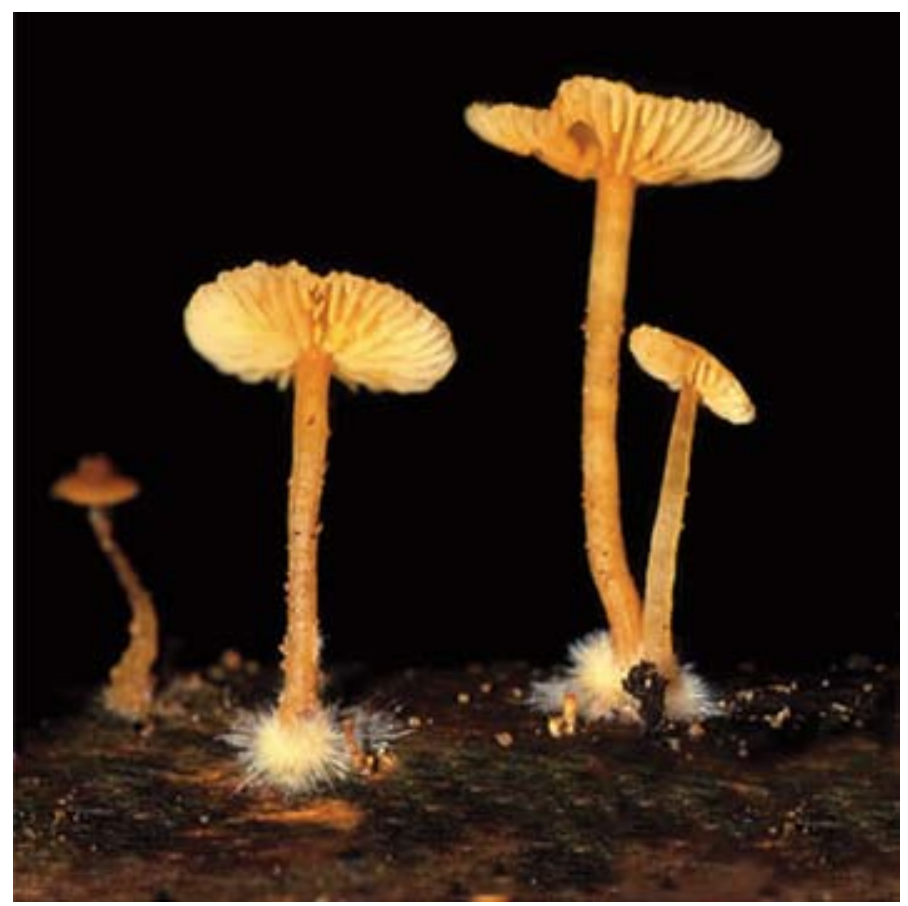

Fig. 2. Basidiomas de Gymnopilus tuxtlensis en la región de El Tuito, Jalisco.

Venezuela, Estado Guarico, aprox. $45 \mathrm{~km} \mathrm{S-SW} \mathrm{de} \mathrm{Calabozo,} \mathrm{Fondo} \mathrm{Pecuario}$ Masaguaral, agosto 3, 1980, C. Ovrebo 1182-A (F); agosto 17, 1980, C. Ovrebo $1215-A(\mathrm{~F}-1059160)$.

Comentarios. Gymnopilus tuxtlensis es una especie muy distintiva por la combinación de sus características. Cuando los basidiomas son jóvenes y con las láminas amarillas, recuerda más un representante de Tricholomataceae, semejante a Xeromphalina, por su tamaño, esbeltez, fragilidad, color y transparencia del píleo; es hasta cuando maduran, que las láminas toman el típico color ferruginoso de Gymnopilus. Otros rasgos particulares son la presencia de la base estrigosa de color amarillo-mostaza y el píleo mamiforme o umbilicado. Micromorfológicamente se caracteriza por el tamaño de sus esporas, más grandes que el término medio de las especies del género, que va de 6-8 $\mu \mathrm{m}$, por sus basidios más cortos, subhimenio celular, queilocistidios pequeños, y en particular por los pleurocistidios grandes, con ápices capitados a moniliformes. En la descripción original (Guzmán-Dávalos, 1994) se indicó la ausencia de caulocistidios; sin embargo, éstos están presentes, aunque 
son muy escasos en el material tipo. También se mencionó que los fragmentos de lámina no liberaban pigmento amarillo en $\mathrm{KOH}$, lo cual es cierto para el tipo, pero no para la mayoría de los demás ejemplares estudiados.

Es difícil encontrar especies afines a G. tuxtlensis. En la descripción original, Guzmán-Dávalos (1994) mencionó que este hongo está relacionado con G. subbellulus Hesler, G. hemipenetrans Guzm.-Dáv. y G. radicicola Singer; sin embargo, estos tres son bastante diferentes de G. tuxtlensis. El más cercano es G. subbellulus por el tamaño y color del basidioma, pero difiere en crecer en la madera de coníferas, tener esporas más pequeñas y presentar pileocistidios (Hesler, 1969). Por otro lado, Rees et al. (1999) compararon a G. tyallus Grgur., una especie australiana, con $G$. tuxtlensis, por el basidioma pequeño y basidiosporas de tamaño similar. Las dos se distinguen principalmente porque G. tyallus tiene el píleo seco, no higrófano y los cistidios lecitiformes a tibiformes.

Como la mayoría de los componentes en el género, G. tuxtlensis es lignícola, desarrollándose sobre ramas o troncos podridos; sin embargo, el ejemplar GuzmánDávalos 7675 se encontró viviendo sobre estiércol, cerca de otros especímenes típicos sobre madera. Probablemente el crecimiento sobre el excremento haya sido secundario por la cercanía con restos de madera. Sobre el estiércol el hongo también formó el halo estrigoso amarillo en la base del estípite. En todos los casos el píleo es glabro a fibriloso-algodonoso; sólo en algunos basidiomas de Guzmán-Dávalos 7670 se observaron pequeñas escamas de color púrpura o rojizas y en general en éstos el estípite es más corto que en el resto.

Las basidiosporas en el espécimen tipo de G. tuxtlensis (Guzmán 19671) son ligeramente más pequeñas y más elipsoides (Guzmán-Dávalos, 2003) que en el resto de los ejemplares revisados, en los cuales hay cierta tendencia a ser de tamaño mayor y oblongas. En varios casos se observó la presencia de un microporo germinal; las basidiosporas del género por lo común no presentan tal estructura, pero en varias especies, por ejemplo en G. subearlei R. Valenz., Guzmán \& J. Castillo se ha llegado a ver este pequeño poro en el microscopio óptico (Guzmán-Dávalos, datos no publicados). Por otro lado, se han realizado observaciones en el microscopio electrónico de barrido de las esporas de G. subearlei, en las que no se logró detectar ninguna modificación en el ápice de la espora (Guzmán-Dávalos, datos no publicados). Como se mencionó, G. tuxtlensis presenta dos tipos de pleurocistidios: los del tipo 1 son muy escasos y no se vieron en todos los especímenes; los pleurocistidios de tipo 2 en general son muy abundantes y evidentes por su tamaño y forma, característicos de esta especie, pero pueden ser escasos o incluso ausentes, como en Guzmán 19830. Los pleurocistidios del tipo 1 son semejantes a los que se presentan, por ejemplo, en 
G. medius Guzm.-Dáv. (Guzmán-Dávalos, 1994); en cambio los del tipo 2 sólo se han observado en G. tuxtlensis.

El ejemplar Ovrebo 1215-A fue registrado como Gymnopilus croceoluteus Hesler por Ovrebo (1983), y el material Ovrebo 1182-A se encontraba depositado en $\mathrm{F}$ también bajo el nombre de $G$. croceoluteus, especie no relacionada con $G$. tuxtlensis. Gymnopilus croceoluteus tiene un basidioma mucho más grande y robusto que el de G. tuxtlensis, beige a anaranjado, margen no estriado, con trama del píleo entrelazada y crece en madera de coníferas en los estados de Michigan, Florida y Idaho, en Estados Unidos. Parece ser que la única semejanza entre estas dos especies es el píleo higrófano, característica poco usual en el género (Hesler, 1969).

\section{AGRADECIMIENTOS}

Se reconoce el apoyo del Consejo Nacional de Ciencia y Tecnología, proyecto CONACYT-SEP-2003-C02-42957; del Programa de Mejoramiento del Profesorado de Educación Superior a través de su programa de apoyo a ex-becarios, proyecto PROMEP/103.5/03/2580, y de la Universidad de Guadalajara, proyectos P3E 34961 del Cuerpo Académico CA-23 y P3E 34332 de las colecciones del Instituto de Botánica, que permitieron llevar a cabo este trabajo. Cardona y Saldarriaga dan las gracias a la Corporación Académica para el estudio de Patologías Tropicales, al Instituto de Biología y al Comité para el Desarrollo de la Investigación (CODI), todas de la Universidad de Antioquia, por el apoyo para realizar esta contribución. Se agradece a Clark Ovrebo por proporcionar sus notas personales y fotografías de sus materiales de Venezuela.

\section{LITERATURA CITADA}

Guzmán-Dávalos, L. 1994. New species of Gymnopilus (Agaricales, Cortinariaceae) from Mexico. Mycotaxon 50: 333-348.

Guzmán-Dávalos, L. 2002. Tropical brown- and black-spored Mexican agarics with particular reference to Gymnopilus. In: Watling, R., J. C. Frankland, A. M. Ainsworth, S. Isaac y C. H. Robinson (eds.). Tropical Mycology, Vol. I. Macromycetes. CAB International. Wallingford. pp. 61-71.

Guzmán-Dávalos, L. 2003. Type studies of Gymnopilus (Agaricales) I. Mycotaxon 84: 395423. 
Hesler, L. R. 1969. North American species of Gymnopilus. Mycologia Memoir No. 3. Hafner Pub. Co. Nueva York. 117 pp.

Holmgren, P. K., N. H. Holmgren y L. C. Barnett. (eds.). 1990. Index Herbariorum. Part I. The herbaria of the world. 8a. ed. New York Botanical Garden. Nueva York. 663 pp.

Kornerup, A. y J. H. Wanscher. 1978. Methuen handbook of colour. 3a. ed. Eyre Methuen. Londres. $252 \mathrm{pp}$.

Ovrebo, C. L. 1983. New records of fleshy fungi from Venezuela. Mycotaxon 18: 355-356.

Rees, B. J., D. A. Orlovich y P. B. D. Marks. 1999. Treading the fine line between smallstatured Gymnopilus and excentrically stipitate Galerina species in Australia. Mycol. Res. 103: 427-442.

Recibido en julio de 2005.

Aceptado en febrero de 2006. 